\title{
IN VIVO CYCLIC LOADING AS A POTENT STIMULATORY SIGNAL FOR BONE FORMATION INSIDE TISSUE ENGINEERING SCAFFOLDS
}

\author{
Alireza Roshan-Ghias ${ }^{1}$, Alexandre Terrier ${ }^{1}$, Pierre-Etienne Bourban ${ }^{2}$ and Dominique P. Pioletti ${ }^{{ }^{*}}$ \\ ${ }^{1}$ Laboratory of Biomechanical Orthopedics, ${ }^{2}$ Laboratory of Composite and Polymer Technology, EPFL, Lausanne, \\ Switzerland
}

\begin{abstract}
In clinical situations, bone defects are often located at load bearing sites. Tissue engineering scaffolds are future bone substitutes and hence they will be subjected to mechanical stimulation. The goal of this study was to test if cyclic loading can be used as stimulatory signal for bone formation in a bone scaffold. Poly(L-lactic acid) (PLA)/ 5\% $\beta$ tricalcium phosphate $(\beta-\mathrm{TCP})$ scaffolds were implanted in both distal femoral epiphyses of eight rats. Right knees were stimulated ( $10 \mathrm{~N}, 4 \mathrm{~Hz}, 5 \mathrm{~min})$ five times, every two days, starting from the third day after surgery while left knees served as control. Finite element study of the in vivo model showed that the strain applied to the scaffold is similar to physiological strains. Using micro-computed tomography (CT), all knees were scanned five times after the surgery and the related bone parameters of the newly formed bone were quantified. Statistical modeling was used to estimate the evolution of these parameters as a function of time and loading. The results showed that mechanical stimulation had two effects on bone volume (BV): an initial decrease in $\mathrm{BV}$ at week 2, and a long-term increase in the rate of bone formation by $28 \%$. At week 13 , the BV was then significantly higher in the loaded scaffolds.
\end{abstract}

Keywords: Bone tissue engineering, osteogenesis, animal model, mechanical stimulation.

* Address for correspondence:

Dominique Pioletti

EPFL/STI/IBI/LBO

Station 15

CH-1015 Lausanne,

Switzerland

E-mail: dominique.pioletti@epfl.ch
Introduction

The growing need for bone substitutes is the driving motivation for researchers to develop bone scaffolds and improve the process of bone regeneration. The latter has been the focus of many studies and some techniques have been proposed for enhancing osteogenesis inside bone scaffolds, like seeding scaffolds with mesenchymal stem cells (Meinel et al., 2004) or with fetal bone cells (Pioletti et al., 2006), delivering growth factors using scaffolds (Murakami et al., 2002; Richardson et al., 2001) or premineralization in in vitro bioreactors (Cartmell et al., 2003). All of these methods have been successful to some extent, but they have certain disadvantages. For instance, the high cost of the final product in all these techniques is certainly an important issue.

Mechanical stimulation is another potential method for enhancing bone regeneration that can be employed by designing the scaffold and controlling the external load accordingly. It has long been known that mechanical stimulation has regulatory effects on bone cells and architecture of bone (Forwood et al., 1996; Turner et al., 1994). In vitro studies suggest that fluid flow enhances expression of the osteoblastic phenotype (Cartmell et al., 2003; Sikavitsas et al., 2003; Tanaka et al., 2005; Zhao et al., 2007), proliferation of mesenchymal stromal cells (Song et al., 2007) and mineralization (Bancroft et al., 2002; Goldstein et al., 2001; Sikavitsas et al., 2005). However, an excessive amount of fluid flow or cyclic compression may decrease the number of cells or have no osteogenic effect (Cartmell et al., 2003; Rath et al., 2008; van Eijk et al., 2008). Although in vitro studies help us understand the mechanisms of mechanotransduction, the cellular events in in vivo conditions are more complex and greatly unknown. In particular, drilling of bone initiates a bone healing cascade that results in different cellular activities and chemical environment compared to quiescent in vitro conditions.

A frequently used model to study the effect of mechanical stimulation on bone regeneration in vivo is the bone chamber. Using bone chambers, some researchers have reported that mechanical loading enhances bone formation (Guldberg et al., 1997; Lamerigts et al., 2000; van der Donk et al., 2002), and some observed that loading inhibits bone formation (Tagil and Aspenberg, 1999). Duty and coworkers (Duty et al., 2007) used a subcutaneous bone chamber and found that cyclic loading of mesenchymal stem cell seeded scaffolds enhances the mineralization.

In the present study, we hypothesized that mechanical stimulation enhances bone regeneration in scaffold. Distal femoral epiphysis of rat was used as an in vivo model to study the effect of mechanical stimulation on bone formation in a polymeric scaffold. Micro computed 

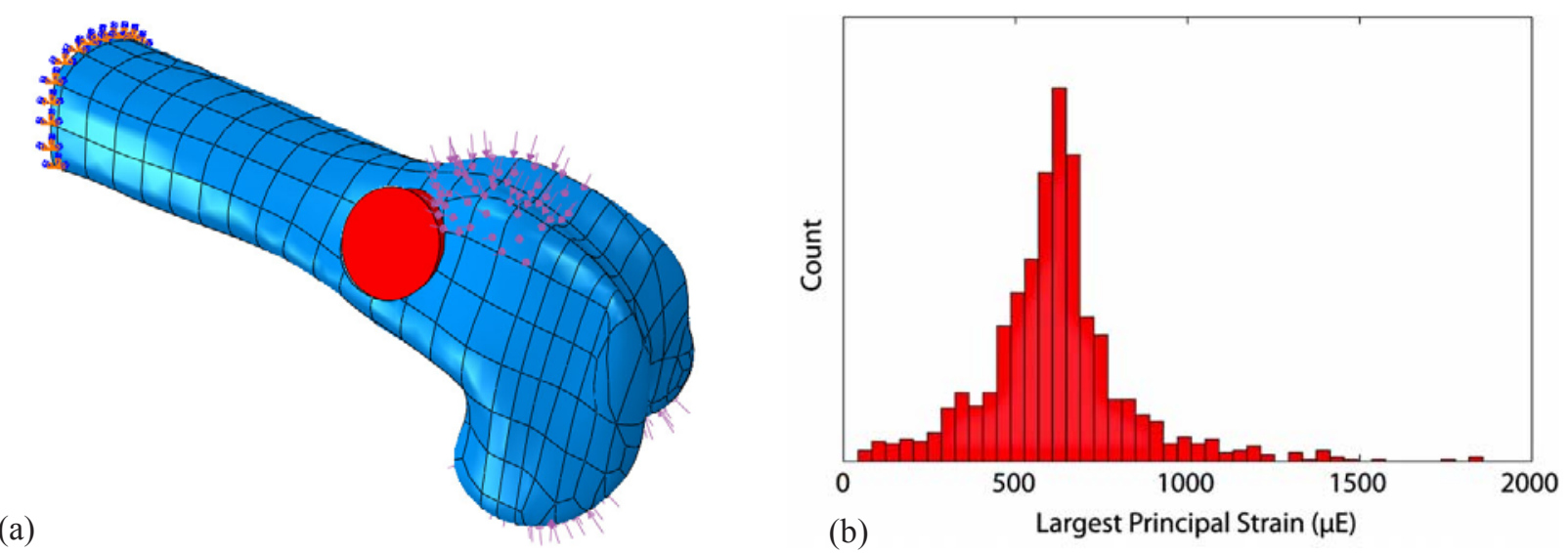

Fig. 1. (a) Distal femoral epiphysis model where bone is in blue and scaffold is in red. The loading is shown by violet arrows on anterior and posterior sides of the distal femur. (b) The histogram of largest principal strain in scaffold. Count represents the number of elements having the same value of strain.

tomography (CT) was used to study bone formation in vivo. Longitudinal analysis was then employed to model and investigate the effect of loading on bone formation.

\section{Materials and Methods}

\section{Finite element modeling}

Finite element modeling was performed to determine the magnitude of load to be applied in in vivo experiment. A rat femur was scanned using a SkyScan 1076 in vivo scanner (SkyScan, Kontich, Belgium), and reconstructed using NRecon and CTAN software (SkyScan) and prepared for meshing using Geomagic (Geomagic, Research Triangle Park, NC, USA). The obtained geometry was imported to ABAQUS (Simulia, Providence, RI, USA) for numerical analysis (Fig. 1a). The scaffold was positioned inside the lateral distal femoral epiphysis based on the feasibility studies done on several rat cadavers. The bone and the scaffold were considered to be linear elastic materials. Mechanical properties of cortical and trabecular bone were obtained from the literature (Hogan et al., 2000). The mechanical properties of the scaffold were previously measured and reported by Blecha (Blecha, 2007) $(\mathrm{E}=20$ $\mathrm{MPa}, \mathrm{v}=0.3$ ). As the boundary condition, the proximal end of the bone was fixed. Based on the cadaver studies, it was assumed that no slip occurs at the interface between scaffold and bone. Loading orientation was based on the positioning of the leg in the loading device. Different magnitudes of load were applied to the anterior and posterior parts of distal femur and the average largest principal strain inside the scaffold was calculated. It was found that $10 \mathrm{~N}$ of loading results in strain of $622 \pm 220 \mu \varepsilon$ inside the scaffold, which is close to physiological strains in human tibia while walking (Burr et al., 1996). Fig. 1b shows the histogram of the distribution of strain inside the scaffold.

\section{Scaffold fabrication}

The processing of foams, as well as analyses of scaffold morphology, was carried out as described elsewhere (Mathieu et al., 2006). In short, poly(L-lactic acid) (PLA) pellets and $\beta$-tricalcium phosphate $(\beta$-TCP) ceramic powder ( $5 \%$ weight) were mixed, and further meltextruded using a micro-compounder. Foaming was then carried out with supercritical $\mathrm{CO}_{2}$, in a custom made highpressure chamber. Once $\mathrm{CO}_{2}$ was dissolved in PLA, foaming was achieved by sudden gas release, which induces bubble nucleation and their growth. Finally the controlled cooling till room temperature fixes the porous structure. The in vitro biocompatibility of the obtained scaffold has been verified (Montjovent et al., 2005) as well as its in vivo performance in rat (Montjovent et al., 2008; Montjovent et al., 2007). Cylindrical scaffolds (3 $\mathrm{mm}$ in diameter and height) were cored from the foam and sterilized using ethylene oxide gas. Prior to surgery, scaffolds were perfused with phosphate-buffered saline (PBS) to remove the air bubbles trapped inside.

\section{Surgical procedure}

Both distal femoral epiphyses of eight female Wistar rats (weight 245-250 g) were operated (Veterinary Authority from the Canton of Vaud, authorization No. 2140) following a protocol already used in our laboratory (Montjovent et al., 2008; Peter et al., 2006). The animal was anesthetized using Isoflorane gas and both legs were shaved and sterilized. Five $\mathrm{ml}$ of Morphasol (Graeub Veterinary Products, Bern, Switzerland) were injected subcutaneously as analgesia. The lateral side of the knee joint was opened and after exposing the distal femur, the location of the hole was marked on the bone based on the distance from the joint and the insertion point of ligaments. The hole ( $3 \mathrm{~mm}$ in diameter and depth) was made using a hand drill and the scaffold was implanted inside the hole. Finally, the surrounding was washed with saline, the muscles were sutured, and the skin was closed using surgical clips (Stoelting, Wood Dale, IL, USA). No antibiotic was given after the surgery, but to reduce the pain, Dafalgan (Bristol-Myers Squibb, New York, NY, USA) was administered in water for 3 days post-surgery.

\section{Mechanical stimulation}

Fig. 2 shows the timeline of the experiment. Three days after the surgery, the loading of the knee started using a 


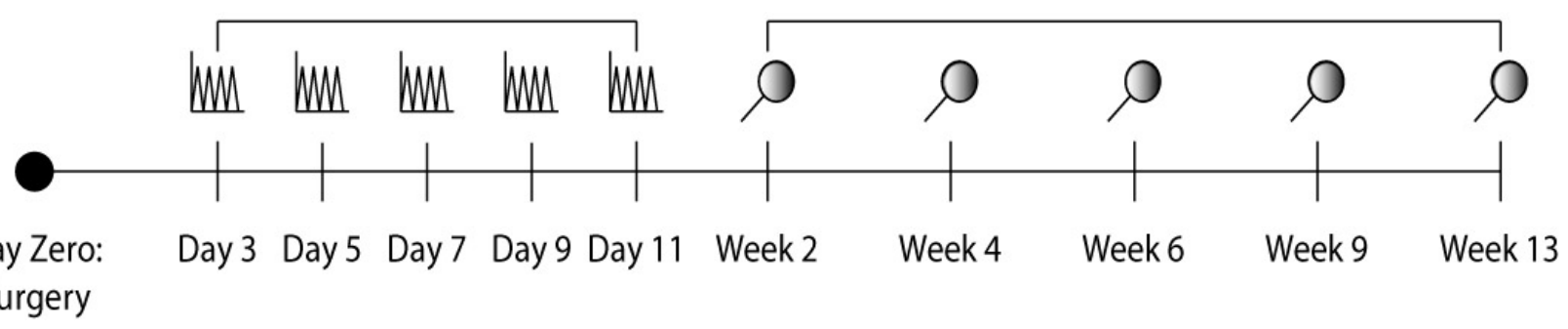

Fig. 2. The timeline of the in vivo experiment.

compression machine previously developed in our laboratory (Stadelmann et al., 2007) (Fig. 3a). The right leg of each animal was loaded and the left leg was kept unloaded as control. The animals were anesthetized by Isoflorane gas for the loading period. The knee and the heel of the right leg were placed in the grip of the machine and the loading was applied as a triangle waveform with amplitude of $10 \mathrm{~N}$ at $4 \mathrm{~Hz}$ for 5 minutes. The loading was done five times, every two days.

\section{Bone formation evaluation}

The bone formation was quantified using a Skyscan 1076 in vivo micro-CT scanner (Skyscan) at 5 time points: 2, 4, 6, 9, and 13 weeks after the surgery. Animals were kept under anesthesia by Isoflorane during the scanning. Each leg was scanned separately along with two hydroxyapatite phantoms with densities of 0.25 and 0.75 g.cm $\mathrm{cm}^{-3}, 8 \mathrm{~mm}$ in diameter (Gloor Instruments, Uster, Switzerland) and a tube of water for later calibration of bone mineral density (BMD) (Fig. 3b). The legs were stretched and confined using tape to isolate the knee joint. All animals were scanned using the same parameters $(18 \mu \mathrm{m}, 80 \mathrm{kV}, 124$ $\mu \mathrm{A}, 1 \mathrm{~mm}$ Al filter, $600 \mathrm{~ms}$ exposure time, $14 \mathrm{~min}$ of scanning) at all time points. The local absorbed dose rate is reported to be $0.043 \mathrm{~Gy} / \mathrm{min}$ for hindlimb knee of rat in identical scanning conditions to ours (data provided by SkyScan). Thus, a 14 min scan will result in radiation dose of $0.6 \mathrm{~Gy}$. The reconstruction and analysis was done using NRecon and CTan software (Skyscan), respectively. A second order polynomial correction algorithm was used to reduce beam-hardening effect for all samples. Since the scaffold is undistinguishable inside the hole, the region of interest (ROI) was selected as the volume of the hole. The CT numbers were first converted to Hounsfield unit (HU) using scans of water tubes, and then to bone mineral density (BMD) using phantoms. Based on visual inspections of tomograms, a threshold value of $0.5 \mathrm{gr} / \mathrm{cm}^{3}$ was chosen to segment bone in the scaffold. Accordingly, bone volume (BV), BMD, interception surface (IS), and trabecular thickness (Tb.Th) were measured for each ROI. Interception surface is the surface of the ROI intercepted by solid binarised objects, that is, the part of the volume of interest boundary surface that runs through solid objects.

\section{Statistical analysis}

Linear mixed-effect modeling was used to model the evolution of BV, BMD, IS, and Tb.Th as a function of time and loading (Diggle et al., 2002). The linear model can be written as

$$
\mathrm{y}_{\mathrm{ijk}}=\left(\beta_{0}+\mathrm{b}_{0 \mathrm{i}}\right)+\left(\beta_{1}+\mathrm{b}_{1 \mathrm{i}}\right) \mathrm{F}_{\mathrm{j}}+\left(\beta_{2}+\mathrm{b}_{2 \mathrm{i}}\right) \mathrm{T}_{\mathrm{k}}+\beta_{12} \mathrm{~F}_{\mathrm{j}} \mathrm{T}_{\mathrm{k}}+\varepsilon_{\mathrm{ijk}}
$$

where $i=1,2,3, \ldots, 8$ is the number of rats, $j=0,1$ is the index for control and loaded groups, respectively, and $k=1,2,3, . ., 5$ is the index for time points. $\beta_{0}, \beta_{1}, \beta_{12}, \beta_{2}$ are the fixed-effect variables, $b_{0 i}, b_{1 i}, b_{2 i}$ are the random-effect variables, and $\varepsilon_{i j k}$ is the within-subject error term.

$\beta_{0}$ is a constant, $\beta_{1}$ represents the time-independent effect of loading, $\beta_{2}$ the loading-independent effect of time, and $\beta_{12}$ the time-dependent effect of loading. $\beta_{0 i}, \beta_{1 i}, \beta_{2 i}$ are the corresponding associated random effects. $y_{i j k}$ is the response (BV, BMD, IS, or Tb.Th), $F_{j}$ is a dummy variable which is 0 for control and 1 for loading group, and $T_{k}$ is the time of measurement in days after the surgery. It is assumed that $b_{i}=\left(b_{0 i}, b_{1 i}, b_{2 i}\right)^{T}$ are independent and identically distributed with a $N\left(0, \sigma^{2} I\right)$ distribution, where $\sigma^{2} \mathbf{I}$ represents the covariance matrix for random effects. Furthermore, we assume that the $\varepsilon_{i j k}$ are independent and identically distributed with a $N\left(0, \sigma^{2}\right)$ distribution, independent of the $b_{i}$. Restricted maximum likelihood method was used for parameter estimation (Pinheiro and Bates, 2004).

Repeated measures analysis of covariances (ANCOVA) was used to evaluate the differences between the control and loaded groups. Differences between means at the $5 \%$ and $1 \%$ confidence level $(p<0.05$, and $p<0.01)$ were considered statistically significant and highly significant, respectively. All statistical analyses were done in S-PLUS (Tibco, Palo Alto, CA, USA).

\section{Results}

No complication occurred during or after the surgeries and all animals could use their legs immediately after the surgery. Implanting scaffold was successful except in three legs. In one case, the scaffold was loose inside the left leg resulting in almost no bone formation inside. In another rat, the scaffolds did not completely fit inside both holes resulting in significantly lower bone formation. Therefore, these two animals were put aside from the experiment. 

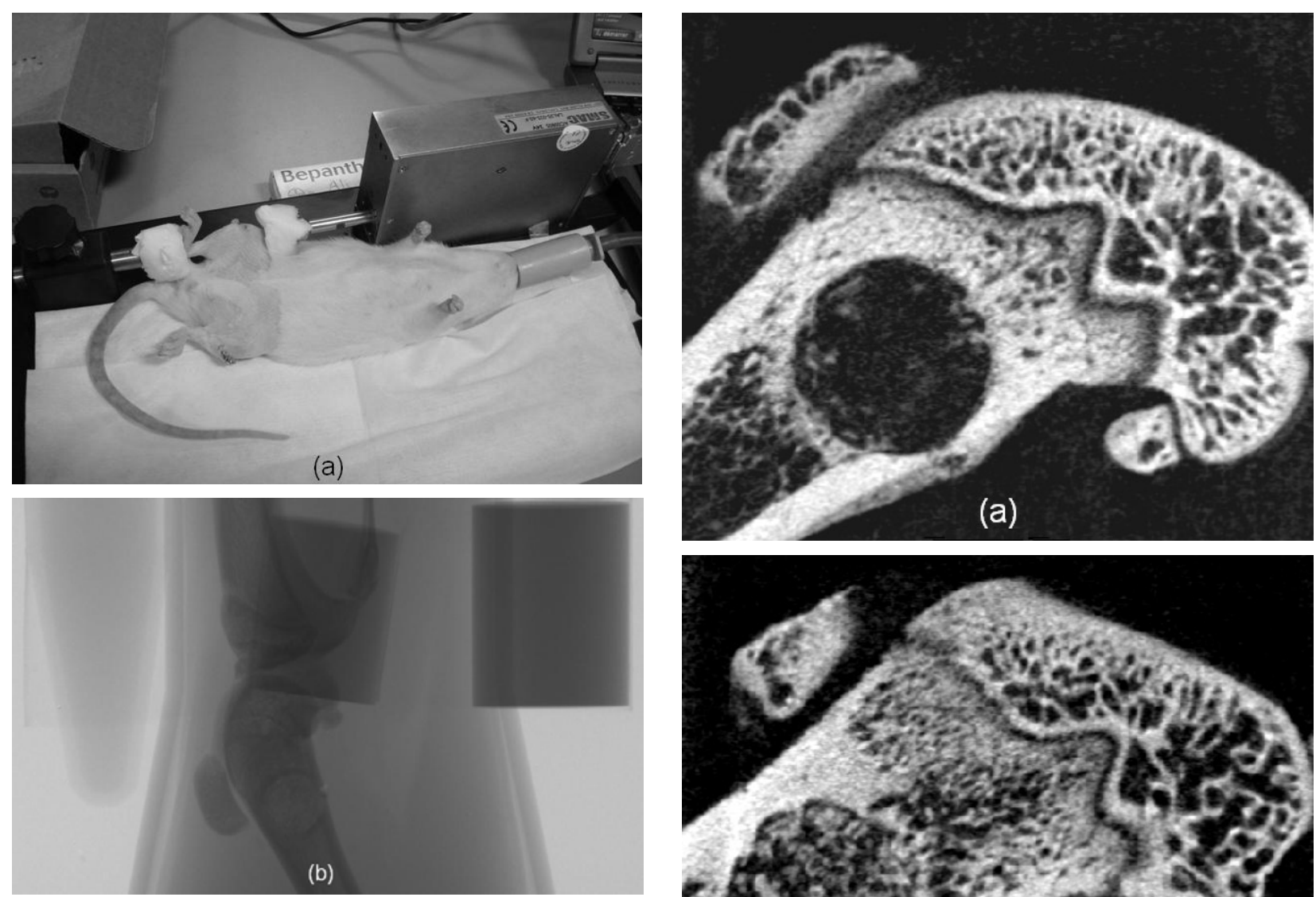

Fig. 3. (a) Anesthetized rat positioned in the loading machine, (b) x-ray of knee joint, two phantoms, and a tube of water.

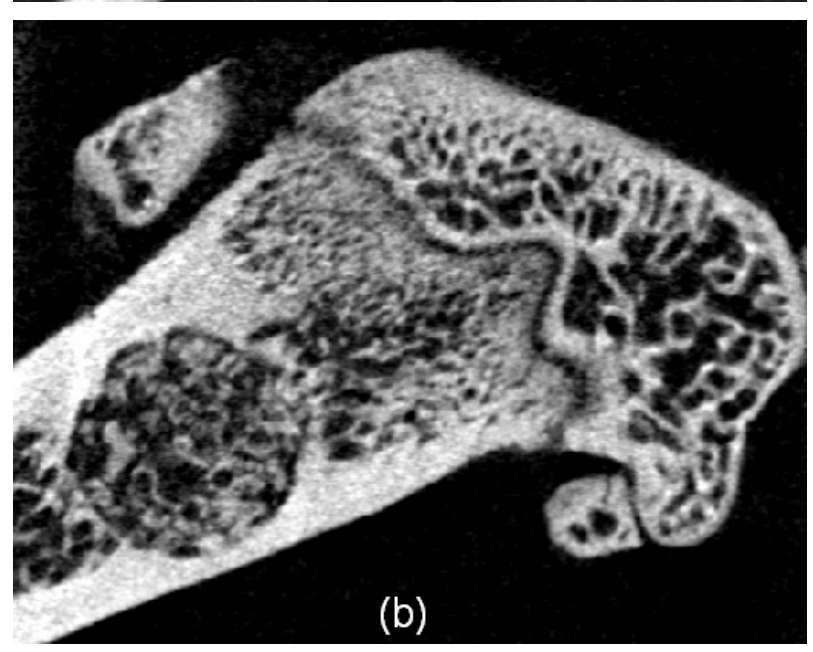

Fig. 4. CT slice of a loaded distal femoral at (a) 2 weeks, (b) 13 weeks. Note that both figures are from the same leg of the same animal and the change in the scaffold position is due to the growth of the animal during 11 weeks.

Table 1. Linear mixed-effect modeling of bone parameters

\begin{tabular}{|l|c|c|c|c|c|c|c|c|c|c|c|c|}
\hline & \multicolumn{4}{|c|}{$\beta 0$} & \multicolumn{4}{c|}{$\beta 1$} & \multicolumn{3}{c|}{$\beta 2$} & \multicolumn{3}{c|}{$\beta 12$} \\
\cline { 2 - 15 } & Mean & SE & $p$-value & Mean & SE & $p$-value & Mean & SE & $p$-value & Mean & SE & $p$-value \\
\hline BV & 0.69 & 0.22 & $<0.0001$ & -0.81 & 0.35 & 0.02 & 0.083 & 0.0056 & $<0.0001$ & 0.023 & 0.0052 & 0.0001 \\
\hline BMD & 0.69 & 0.012 & $<0.0001$ & -0.036 & 0.016 & 0.03 & 0.0017 & 0.0002 & $<0.0001$ & 0.00036 & 0.00025 & 0.1661 \\
\hline IS & 8.63 & 0.91 & $<0.0001$ & -2.25 & 1.26 & 0.08 & 0.12 & 0.017 & $<0.0001$ & 0.016 & 0.021 & 0.47 \\
\hline Tb.Th & 95.58 & 6.26 & $<0.0001$ & -19.48 & 9.81 & 0.05 & 0.97 & 0.1 & $<0.0001$ & 0.37 & 0.1 & 0.0005 \\
\hline
\end{tabular}

Mean value and standard error (SE) of $\beta_{0}, \beta_{1}, \beta_{2}, \beta_{12}$ for BV, BMD, IS, and Tb.Th as well as the $p$-value from the ANCOVA test for coefficients of each parameter are given. The $p$-values lower than 0.05 are marked with light gray background. BV, BMD, IS, and Tb.Th represent bone volume, bone mineral density, interception surface, and trabecular thickness respectively. $\beta_{0}$ is a constant, $\beta_{1}$ represents the time-independent effect of loading, $\beta_{2}$ the loading-independent effect of time, and $\beta_{12}$ the time-dependent effect of loading. 


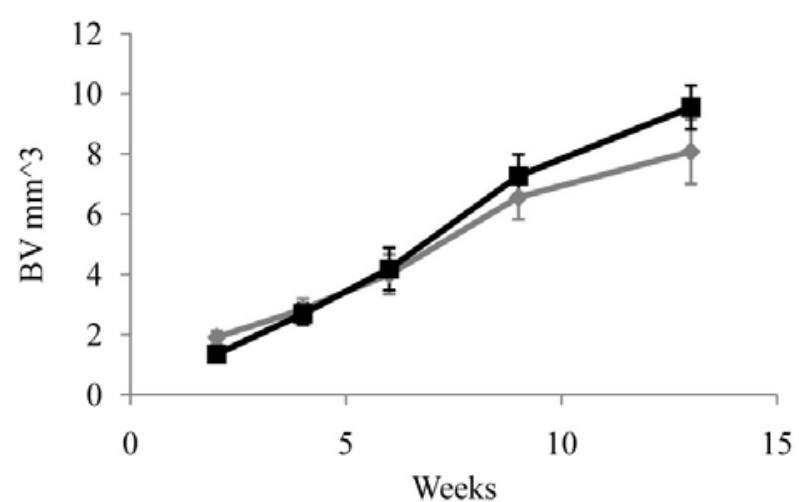

(a)

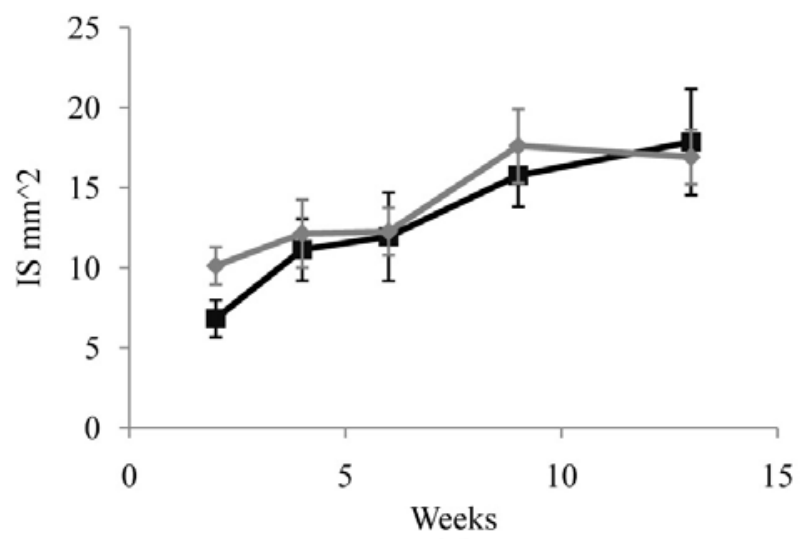

(c)

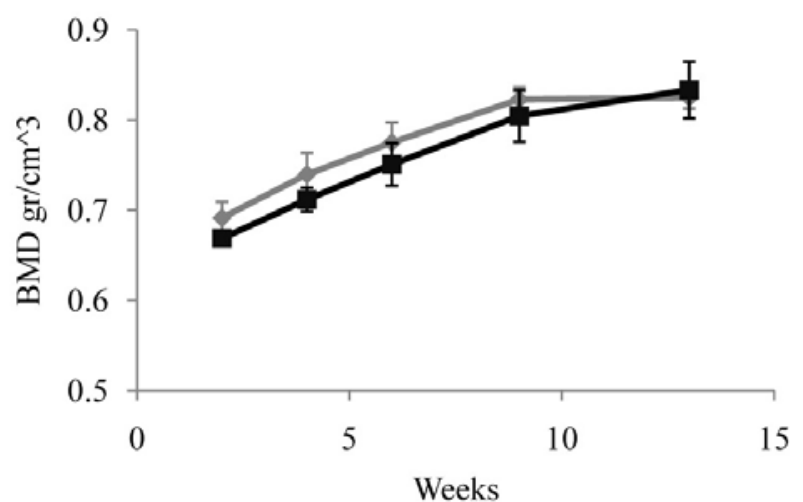

(b)

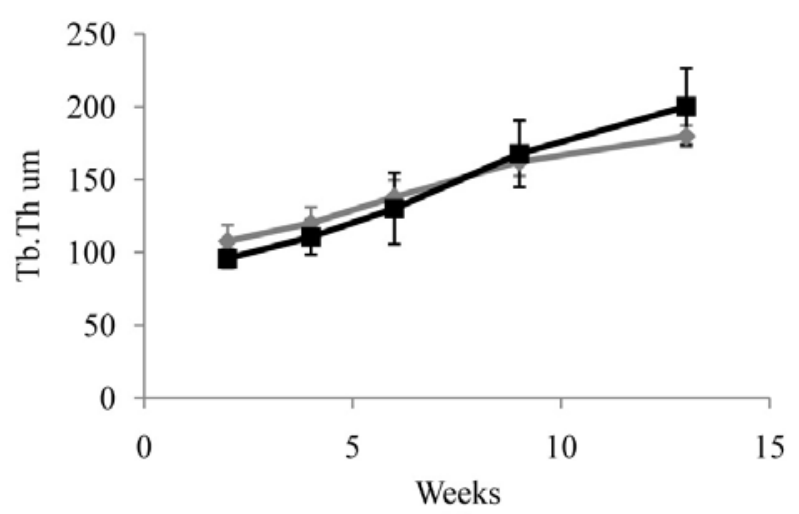

(d)

Fig. 5. The evolution of (a) BV, (b) BMD, (c) IS, and (d) Tb. Th over time for control (light gray - $>_{-}$) and loaded (black - -) groups. All data are presented as mean $\pm 95 \%$ confidence interval.

Fig. 4 shows a CT slice of a loaded distal femoral epiphysis at 2 weeks and 13 weeks after the surgery. Already at 2 weeks, small amount of bone can be seen inside the scaffold that is mainly formed at the outermost part of the scaffold. We also can see callus formation around the hole in all cases at 2 weeks, which is mineralized at 4 weeks and shrunk at later time points. At 13 weeks, the new bone invades the interior of scaffold and is well spread.

Equation (2) is the estimated linear model for bone parameters as a function of time and loading

$$
\mathrm{Y}=\left(\beta_{0}+\beta_{1} \mathrm{~F}\right)+\left(\beta_{2}+\beta_{12} \mathrm{~F}\right) \mathrm{T}
$$

where $Y$ is the estimated bone parameter, $F$ is the dummy variable of loading which is 0 for control and 1 for loaded groups, and $\mathrm{T}$ is time in days. The effect of loading in eqn 2 is twofold: $\beta$, which is the effect of loading on $\mathrm{Y}$ as an intercept, and $\beta_{12}$, which is the effect of loading on the rate of change of $Y$.

Table 1 summarizes the estimated $\beta_{0}, \beta_{1}, \beta_{12}, \beta_{2}$ for $\mathrm{BV}, \mathrm{BMD}$, IS, and Tb.Th. The effects of loading and time on the different bone parameters are individually presented below.

Fig. 5a shows that BV in the loaded group is initially lower compared to the control group. This is reflected in the negative sign of the intercept, $\beta$. On the other hand, at 13 weeks, the loaded group has a higher bone volume (BV). Therefore, the rate of bone formation is higher in the loaded group ( $p$-value $=0.0001)$. Looking at the mean values of $\beta_{12}$ and $\beta_{2}$, we conclude that the loading has enhanced the rate of bone formation by $28 \% \pm 7 \%$.

Looking at Fig. 5b, we see that loading induced a shift in BMD in comparison to control group. The linear mixedeffect model showed that the loading has significantly ( $p$ value $=0.03$ ) reduced the BMD of newly formed bone inside scaffold by $5 \% \pm 2 \%$.

Loading has no significant effect on intercept and rate of change of IS. Only at the first time point, a distinct difference between loaded and control group can be seen (Fig. 5c).

Fig. 5d shows that the loaded group has lower Tb.Th compared to the control group at two weeks ( $p$-value $=$ $0.05)$. However, the rate of change of $\mathrm{Tb}$.Th is higher in the loaded group $(38 \% \pm 10 \%)$, which is reflected, in a highly significant effect of $\beta_{12}$.

\section{Discussion}

The goal of this study was to investigate the effect of mechanical stimulation on bone formation inside tissue engineering scaffolds. A new in vivo model was developed using distal femoral epiphysis of rat where a biocomposite polymeric scaffold was implanted in and later stimulated using an external loading device. In vivo micro-CT was used to measure newly formed bone parameters inside scaffold at several time points. To the best of the authors' 
knowledge, this is the first in vivo model to study the effect of mechanical stimulation on scaffold that is implanted inside bone.

As bone tissue engineering is relatively a new subject, there are only few studies done on the effect of loading on bone formation inside scaffold. Duty and coworkers (Duty et al., 2007) loaded a pre-seeded polymeric scaffold in a subcutaneous bone chamber and studied the effect of cyclic loading on mineralization. The advantage of the distal femur model over the subcutaneous model of Duty (Duty et al., 2007) is that the scaffold is implanted inside bone. This is critical because by implanting the scaffold inside the bone, a process of healing is initiated inside the scaffold. In this process, numerous growth factors are secreted by blood cells that cause mesenchymal stromal cells to migrate from the periosteum and bone marrow into the scaffold and differentiate into osteoblasts (Davies, 2000; Muschler et al., 2004). This process is absent in a subcutaneous model.

The PLA/ 5\% $\beta$-TCP biocomposite scaffold was implanted without any prior cell seeding inside the scaffold. Fig. 4a shows that even two weeks after implantation, bone formation can be seen in the scaffold. This is in accordance with the results of Vehof (Vehof et al., 2002), who reported the presence of bone nine days post surgery in porous scaffolds. Moreover, already at two weeks, we observed a significant amount of compact bone around the scaffold. This is not due to the compaction of trabecular bone caused by drilling, as the results of an ex-vivo drilling and scanning showed almost no compaction of trabecular bone around the hole (data not shown). Therefore, this perimetric compact bone is formed during the first two weeks. Although not studied here, this perimetric bone formation may have important effects on bone formation inside the scaffold. A possible effect can be stress shielding of the scaffold when it is subjected to loading, or delaying the bone healing inside scaffold due to the blocking of vasculature growth into the scaffold.

Live micro-CT scanning enabled us to study the same animals at 5 time points and hence reduced the variability and number of animals. All animals were subjected to xray radiation five times, which may raise some concerns about the effect of radiation on bone and surrounding tissue. As both control and loaded legs were scanned, the possible effect of radiation would be the same for all samples and cancelled by comparing loaded and control groups together. Moreover, it has been shown that in vivo micro-CT radiation has no significant effect on structural parameters of rat tibias and bone marrow cells after eight weekly scans (Brouwers et al., 2007). In the study of Brouwers, the radiation dose for the 35 minutes scan is reported to be 0.9 Gy compared to the 0.6 Gy in our case. Moreover, in our study we scanned the animals 5 times and the duration of rest between each scan was at least two weeks. Therefore, we can conclude that the radiation doses used in the present study had no significant effects on the obtained results.

Finite element modeling was developed to determine the magnitude of loading in the in vivo experiment. We targeted loads that result in physiological strains inside the scaffold, based on the fact that scaffolds, as bone substitutes, are likely to be subjected to physiological loads in clinical applications. The result of the finite element model showed that $10 \mathrm{~N}$ of loading resulted in strains around $620 \mu \varepsilon$, which is close to the strains induced in human tibia while walking (Burr et al., 1996). A pilot study revealed that loads higher than $10 \mathrm{~N}$ were painful for the animals. Therefore, we chose $10 \mathrm{~N}$ as a loading magnitude for the in vivo study. The resulting strain within the scaffold is 20 times lower than the strain on the scaffold in the subcutaneous model of Duty (Duty et al., 2007). Due to the difference in geometry and architecture of the scaffolds, these values cannot be compared directly.

The highly significant effects of intercept and time on all parameters shows the goodness of the fit of equation 1 , which was expected due to the almost linear evolution of the bone parameters in time (Fig. 5 (a)-(d)). In contrast to the presented data, one would expect that the intercept be zero for all parameters, since scaffolds were empty at time zero. This shows that the linear model is not suitable for predicting the early stage of bone formation (0-2 weeks).

As can be seen in Fig. 5a, loading had a negative effect on bone formation immediately after the loading. This is an interesting finding, which is in contrast with the common belief on early load bearing of bone after surgery (Claes et al., 2009). Nevertheless, the mechanical stimulation increased the rate of bone formation, which finally resulted in a higher bone volume in the loaded group. The reason for the early negative effect is not clear, but it might be due to the high stresses that were applied to the interface of the scaffold-bone, as the interception surface is also lower in loaded group at 2 weeks. A similar result has been reported in fracture healing studies where early loading of the fracture site resulted in a lower volume of callus formation in mice with osteotomized tibias (Gardner et al., 2008). Using histological methods, it was found that the amount of osteoid in loaded calluses was significantly higher than in the control group. If the same phenomenon is true for the stimulated scaffolds, it may explain the later increase in bone volume. However, if the loading magnitude had been higher, the damage would have been more extensive and that would have resulted in less bone formation at later time points. Therefore excessive loading at early stages might have had a more long-term negative effect. However, on the other hand, starting the loading later on, or using moderately lower early load may resolve this problem. Further studies should be done to examine this hypothesis.

An interesting result of the present study is that only five sessions of stimulation, each lasting 5 minutes only, resulted in a significant long-term change in the rate of bone formation. To the best of the authors' knowledge, this phenomenon has never been reported for scaffold in bone. It has been reported before in bone remodeling studies where cyclic loading enhanced the rate of bone formation (Chow et al., 1993; Turner et al., 1995). The mechanism by which mechanical stimulation affects the rate of bone formation was not studied here, but it can be due to the effect of loading-induced fluid flow on differentiation of mesenchymal stem cells (Meinel et al., 2004; Sikavitsas et al., 2005; Zhao et al., 2007). 
BMD of the newly formed bone shows a constant increase in time, which implies that the bone is getting mature. The linear mixed effect model shows that the BMD of the loaded group is significantly lower than that of the control group ( $p$-value $<0.05$ ). The probable reason is that due to the higher rate of bone formation, the amount of osteoid bone is also higher in the loaded group, as was seen in fracture healing (Gardner et al., 2006). Therefore, presence of more immature bone decreases the mean BMD of the newly formed bone inside the scaffold. The interception surface is a measure of the connectivity of exterior bone and scaffold. As Fig. 4a shows, at two weeks after surgery, most of the bone is formed at the perimeter of the scaffold. Since at this time point the bone volume is lower in loaded group, the interception surface is also smaller. However, no significant difference was found between linear mixed-effect models of the loaded and control group. This implies that loading does not affect the interface between bone and scaffold in the long run. Trabecular thickness is a structural parameter for the newly formed bone. It is well-known that osteoblasts lay down bone layer by layer in the process of bone healing (Davies 2000) and Tb.Th represents the mean value of the thickness of the newly formed bone inside scaffold. The higher rate of change of $\mathrm{Tb}$. Th shows that the process of laying bone by osteoblasts is enhanced by mechanical stimulation.

Using distal femoral epiphysis of rat as an in vivo model has certain advantages and disadvantages. On the downside, due to the size of the femur, the size and shape of the implanted scaffold are limited. Moreover, the magnitude of load applied to the scaffold is also limited, because excessive loads may result in damage to the knee joint. On the other hand, the small size of the rat enabled us to scan the scaffolds in vivo in a longitudinal study and track the changes in bone formation more precisely by reducing the sources of variation between rats and scaffolds. Another advantage of this model is that the scaffold is implanted inside trabecular bone directly, a situation similar to clinical applications.

More research is needed to characterize the effect of mechanical stimulation on bone formation in a scaffold for clinical application. There are many parameters involved in the effect of loading, and changing them may have positive or negative effects on bone formation. The magnitude and frequency of loading, for instance, are important parameters, which certainly affect the bone formation. The duration and the pattern of loading may also have important role. Moreover, the architecture and material properties of the scaffold and cell-seeding are other important aspects that possibly have interactions with the effect of the mechanical stimulation. The distal femoral epiphysis of the rat seems to be a suitable in vivo model for studying these different loading parameters on the bone formation in scaffold.

\section{Conclusions}

Supporting load is an important function of bone, which inevitably will be inherited by bone scaffolds. In vitro studies suggest that mechanical stimulation has an important role in the fate and activities of the cells inside scaffold. We employed the distal femoral epiphysis of rat with an external loading device to investigate the mechanical effect of loading on bone formation in a polymeric scaffold. We observed that on one hand, early two-week loading inhibits bone formation initially, but on the other hand it enhanced the rate of bone formation by $28 \%$ for the next 11 weeks. At the end of 13 weeks, the volume of newly-formed bone was higher in loaded scaffolds compared to the non-loaded scaffolds. The strain sustained by the scaffold was estimated to be $620 \mu \varepsilon$, at a frequency of $4 \mathrm{~Hz}$. More studies are needed to fully characterize the effect of loading, and the distal femoral epiphysis of rat seems to be a suitable model for this purpose. This study showed that mechanical stimulation can be a potent enhancing signal for in vivo bone formation in tissue engineering scaffolds.

\section{Acknowledments}

This project was supported by a SNSF grant (\#205320121893) and the Inter-institutional Center for Translational Biomechanics EPFL-CHUV-DAL. Dr. Vahid Partovinia (EPFL) is acknowledged for his scientific advice regarding statistical analysis.

\section{References}

Bancroft GN, Sikavitsas VI, van den Dolder J, Sheffield TL, Ambrose CG, Jansen JA, Mikos AG (2002) Fluid flow increases mineralized matrix deposition in $3 \mathrm{D}$ perfusion culture of marrow stromal osteoblasts in a dose-dependent manner. Proc Natl Acad Sci USA 99: 12600-12605.

Blecha LD (2007) Theoretical and Experimental Multiscale Study of an Artificial Bone Construct: from Tibial Osteotomy to Cell/Fluid Interaction. Thesis, Ecole Polytechnique Federale de Lausanne.

Brouwers JE, van Rietbergen B, Huiskes R (2007) No effects of in vivo micro-CT radiation on structural parameters and bone marrow cells in proximal tibia of wistar rats detected after eight weekly scans. J Orthop Res 25: 1325-1332.

Burr DB, Milgrom C, Fyhrie D, Forwood M, Nyska M, Finestone A, Hoshaw S, Saiag E, Simkin A (1996) In vivo measurement of human tibial strains during vigorous activity. Bone 18: 405-410.

Cartmell SH, Porter BD, Garcia AJ, Guldberg RE (2003) Effects of medium perfusion rate on cell-seeded three-dimensional bone constructs in vitro. Tissue Eng 9: 1197-1203.

Chow JW, Jagger CJ, Chambers TJ (1993) Characterization of osteogenic response to mechanical stimulation in cancellous bone of rat caudal vertebrae. Am J Physiol 265: E340-347.

Claes L, Blakytny R, Göckelmann M, Schoen M, Ignatius A, Willie B (2009) Early dynamization by reduced fixation stiffness does not improve fracture healing in a rat femoral osteotomy model. J Orthop Res 27: 22-27. 
Davies JE (2000) Bone Engineering. Em squared Inc., Toronto, Canada.

Diggle PJ, Heagerty P, Liang K-Y and Zeger SL (2002) Analysis of Longitudinal Data, Oxford University Press, Oxford, UK.

Duty AO, Oest ME, Guldberg RE (2007) Cyclic mechanical compression increases mineralization of cellseeded polymer scaffolds in vivo. J Biomech Eng 129: 531539.

Forwood MR, Owan I, Takano Y, Turner CH (1996) Increased bone formation in rat tibiae after a single short period of dynamic loading in vivo. Am J Physiol Endocrinol Metab 33: E419-E423.

Gardner MJ, van derMeulen MC, Demetrakopoulos D, Wright TM, Myers ER, Bostrom MP (2006) In vivo cyclic axial compression affects bone healing in the mouse tibia. J Orthop Res 24: 1679-1686.

Gardner MJ, Ricciardi BF, Wright TM, Bostrom MP, vanderMeulen MC (2008) Pause insertions during cyclic in vivo loading affect bone healing. Clin Orthop and Relat Res 466: 1232-1238.

Goldstein AS, Juarez TM, Helmke CD, Gustin MC, Mikos AG (2001) Effect of convection on osteoblastic cell growth and function in biodegradable polymer foam scaffolds. Biomaterials 22: 1279-1288.

Guldberg RE, Caldwell NJ, Guo XE, Goulet RW, Hollister SJ, Goldstein SA (1997) Mechanical stimulation of tissue repair in the hydraulic bone chamber. J Bone Miner Res 12: 1295-1302.

Hogan HA, Ruhmann SP, Sampson HW (2000) The mechanical properties of cancellous bone in the proximal tibia of ovariectomized rats. J Bone Min Res 15: 284-292.

Lamerigts NMP, Buma P, Huiskes R, Schreurs W, Gardeniers J, Slooff TJJH (2000) Incorporation of morsellized bone graft under controlled loading conditions. A new animal model in the goat. Biomaterials 21: 741747.

Mathieu LM, Mueller TL, Bourban PE, Pioletti DP, Muller R, Manson JAE (2006) Architecture and properties of anisotropic polymer composite scaffolds for bone tissue engineering. Biomaterials 27: 905-916.

Meinel L, Karageorgiou V, Fajardo R, Snyder B, Shinde-Patil V, Zichner L, Kaplan D, Langer R, VunjakNovakovic G (2004) Bone tissue engineering using human mesenchymal stem cells: Effects of scaffold material and medium flow. Ann Biomed Eng 32: 112-122.

Montjovent MO, Mathieu L, Hinz B, Applegate LL, Bourban PE, Zambelli PY, Manson JA, Pioletti DP (2005) Biocompatibility of bioresorbable poly(L-lactic acid) composite scaffolds obtained by supercritical gas foaming with human fetal bone cells. Tissue Eng 11: 1640-1649.

Montjovent MO, Mathieu L, Schmoekel H, Mark S, Bourban PE, Zambelli PY, Laurent-Applegate LA, Pioletti DP (2007) Repair of critical size defects in the rat cranium using ceramic-reinforced PLA scaffolds obtained by supercritical gas foaming. J Biomed Mat Res 83: 41-51.

Montjovent MO, Mark S, Mathieu L, Scaletta C, Scherberich A, Delabarde C, Zambelli PY, Bourban PE, Applegate LA, Pioletti DP (2008) Human fetal bone cells associated with ceramic reinforced PLA scaffolds for tissue engineering. Bone 42: 554-564.
Murakami N, Saito N, Horiuchi H, Okada T, Nozaki K, Takaoka K (2002) Repair of segmental defects in rabbit humeri with titanium fiber mesh cylinders containing recombinant human bone morphogenetic protein-2 (rhBMP-2) and a synthetic polymer. J Biomed Mater Res 62: 169-174.

Muschler GE, Nakamoto C, Griffith LG (2004) Engineering principles of clinical cell-based tissue engineering. J Bone Joint Surg [Am] 86A: 1541-1558.

Peter B, Gauthier O, Laib S, Bujoli B, Guicheux J, Janvier P, van Lenthe GH, Muller R, Zambelli PY, Bouler JM, Pioletti DP (2006) Local delivery of bisphosphonate from coated orthopedic implants increases implants mechanical stability in osteoporotic rats. J Biomed Mat Res 76: 133-143.

Pinheiro JC, Bates DM (2004) Mixed-effect models in S and S-PLUS. Springer, Berlin, Germany.

Pioletti DP, Montjovent MO, Zambelli PY, Applegate L (2006) Bone tissue engineering using foetal cell therapy. Swiss Med Wkly 136: 557-560.

Rath B, Nam J, Knobloch TJ, Lannutti JJ, Agarwal S (2008) Compressive forces induce osteogenic gene expression in calvarial osteoblasts. J Biomech 41: 10951103.

Richardson TP, Peters MC, Ennett AB, Mooney DJ (2001) Polymeric system for dual growth factor delivery. Nat Biotechnol 19: 1029-1034.

Sikavitsas VI, Bancroft GN, Holtorf HL, Jansen JA, Mikos AG (2003) Mineralized matrix deposition by marrow stromal osteoblasts in $3 \mathrm{D}$ perfusion culture increases with increasing fluid shear forces. Proc Natl Acad Sci USA 100: 14683-14688.

Sikavitsas VI, Bancroft GN, Lemoine JJ, Liebschner MA, Dauner M, Mikos AG (2005) Flow perfusion enhances the calcified matrix deposition of marrow stromal cells in biodegradable nonwoven fiber mesh scaffolds. Ann Biomed Eng 33: 63-70.

Song GB, Ju Y, Shen XD, Luo Q, Shi YS, Qin J (2007) Mechanical stretch promotes proliferation of rat bone marrow mesenchymal stem cells. Colloids Surf B 58: 71277.

Stadelmann VA, Hocke JM, Verhelle JR, Pioletti DP (2007) Strain distribution in mice tibia under axial loading. Numerical, experimental models. Comp Meth Biomech Biomed Eng 10 (S1): 80-90.

Tagil M, Aspenberg P (1999) Cartilage induction by controlled mechanical stimulation in vivo. J Orthop Res 17: 200-204.

Tanaka SM, Sun HB, Roeder RK, Burr DB, Turner $\mathrm{CH}$, Yokota H (2005) Osteoblast responses one hour after load-induced fluid flow in a three-dimensional porous matrix. Calcif Tissue Int 76: 261-271.

Turner CH, Forwood MR, Otter MW (1994) Mechanotransduction in Bone - Do Bone-Cells Act as Sensors of Fluid-Flow. FASEB J 8: 875-878.

Turner CH, Owan I, Takano Y (1995) Mechanotransduction in bone: role of strain rate. Am J Physiol 269: E438-442.

van der Donk S, Buma P, Verdonschot N, Schreurs BW (2002) Effect of load on the early incorporation of impacted morsellizedallografts. Biomaterials 23: 297-303. 
van Eijk F, Saris DB, Creemers LB, Riesle J, Willems WJ, van Blitterswijk CA, Verbout AJ, Dhert WJ (2008) The effect of timing of mechanical stimulation on proliferation and differentiation of goat bone marrow stem cells cultured on braided PLGA scaffolds. Tissue Eng Part A 14: 1425-1433.

Vehof JW, Takita H, Kuboki Y, Spauwen PH, Jansen JA (2002) Histological characterization of the early stages of bone morphogenetic protein-induced osteogenesis. J Biomed Mater Res 61: 440-449.

Zhao F, Chella R, Ma T (2007) Effects of shear stress on 3-D human mesenchymal stem cell construct development in a perfusion bioreactor system: Experiments and hydrodynamic modeling. Biotech Bioeng 96: 584-595. 